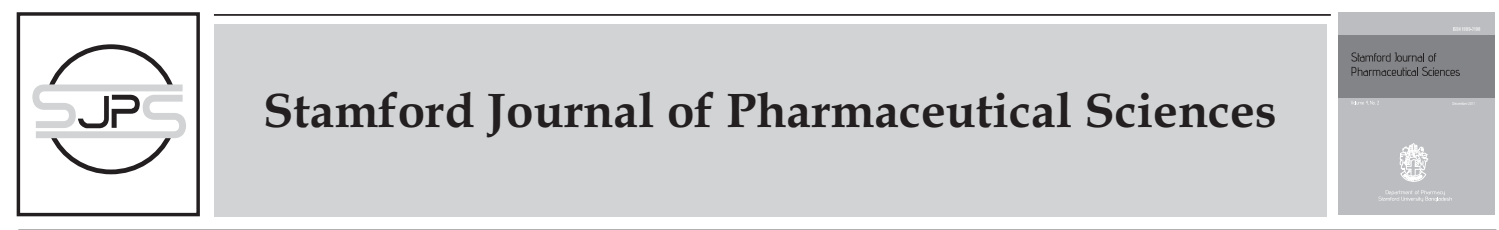

\title{
Dissolution study of Spironolactone by using solid dispersion technique
}

\author{
Irin Dewan, S. M. Ashraful Islam and *Mohammad Shahriar \\ Department of Pharmacy, University of Asia Pacific, Dhanmondi, Dhaka-1209, Bangladesh
}

Original Research Article

\begin{abstract}
The main objective of the current study was to formulate poorly water soluble drug Spirinolactone by using solid dispersion technique in order to achieve a better dissolution rate which would further help in enhancing oral bioavailability. Solid dispersions were prepared using two methods; solvent method and fusion method. Solid dispersion was prepared by using polymers, such as Hydroxy propylymethyl cellulose (HPMC 6cp), Hydroxy propyl cellulose (HPC), Sodium carboxymethylcellulose (Na-CMC), Povidone K12, Povidone K30, Poloxamer 407. Solid dispersions containing Spironolactone with HPC (96.81\%), HPMC $6 \mathrm{cp}$ (93.05\%), Poloxamer 407 (90.84\%) and Na-CMC (89.93\%) provided higher release rate than the release rate of solid dispersion containing only Spironolactone (35.27\%), and Spironolactone with Povidone K12 (76.17\%), Povidone K30 (67.92\%). So the present study revealed that the solid dispersion may be an ideal means of drug delivery system for poorly water soluble drugs. Further study in this field was required to establish these drug delivery systems so that in future it can be used effectively in commercial basis.
\end{abstract}

Keywords: Solid dispersion, Spirinolactone, dissolution, bioavailability.

\section{INTRODUCTION}

Orally administered drugs completely absorb only when they show fair solubility in gastric medium and such drugs shows good bioavailability. Recently more than $40 \%$ NCEs (new chemical entities) developed in Pharmaceutical Industry are practically insoluble in water. These poorly water soluble drugs are allied with slow drug absorption leading to inadequate and variable bioavailability and gastrointestinal mucosal toxicity (Murray et al., 1997).

Oral bioavailability of a drug depends on its solubility and/or dissolution rate, and

*Corresponding Author:

Mohammad Shahriar, Assistant Professor

Department of Pharmacy University of Asia Pacific

House no. 73, Road no. 5A, Dhanmondi

Dhaka-1209, Bangladesh

Telephone: 011-99-844-259

E-mail: shahriar12@yahoo.com dissolution may be the rate determining step for the onset of therapeutic activity. Solid dispersion, which was introduced in the early 1970 s, is essentially a multi-component system, having drug dispersed in and around hydrophilic carrier(s). In order to improve the solubility and bioavailability of poorly water soluble drugs many methods are used. The solid dispersion approach has been widely used for improvement solubility, dissolution rate and hence bioavailability. Solid dispersion (SD) is one of such methods and it involves a dispersion of one or more active ingredients in an inert carrier or matrix in solid state prepared by melting, dissolution in solvent or melting-solvent method (Chiou and Riegelman, 1971). The technique has been used for a wide variety of poorly aqueous soluble drugs such as nimesulide (Babu et al., 2003), ketoprofen (Rogers and Anderson, 1982), tenoxicam (El-Gazayerly, 2000), 
nifedipine (Vippagunta et al., 2002), nimodipine (Babu et al., 2002). SD technology has been successfully been used for improving the solubility of the drugs and hence bioavailability, e.g., tenoxicam, tacrolimus (Yamashita et al., 2003), indomethacin (Makiko et al., 2005), ibuprofen (Loganathanat al., 2000), nilvadipine (Hirasawa et al., 2009).

Therefore, the improvement of drug solubility
MATERIALS AND METHODS

Materials: Spironolactone (Zhejiang Shenzhou Pharmaceutical Comp. Ltd., China), Methanol (MERCK, Germany), Acetone (MERCK, Germany), Distilled water (University Laboratory), HPMC 6cp (Samsung, Korea), HPC (Samsung, Korea), Povidone K30 (F. MARK), Povidone K12 (F. MARK), Poloxamer 407 (BASF), NaCMC (Samsung, Korea), Lactose (Mingel,

Table 1: Formulation for the preparation of Spironolactone solid dispersions using various excipients.

\begin{tabular}{lllllll}
\hline Ingredients & SPL- HPMC & SPL- HPC & SPL-NaCMC & SPL-Pov K12 & SPL-Pov K30 & SPL-Polo \\
\hline Spironolactone & $500 \mathrm{mg}$ & $500 \mathrm{mg}$ & $500 \mathrm{mg}$ & $500 \mathrm{mg}$ & $500 \mathrm{mg}$ & $500 \mathrm{mg}$ \\
Acetone & $5 \mathrm{ml}$ & $5 \mathrm{ml}$ & $5 \mathrm{ml}$ & $5 \mathrm{ml}$ & $5 \mathrm{ml}$ & $5 \mathrm{ml}$ \\
HPMC 6cps & $500 \mathrm{mg}$ & & & & & \\
HPC & & $500 \mathrm{mg}$ & & & \\
NaCMC & & & $500 \mathrm{mg}$ & &
\end{tabular}

Povidone K12

$500 \mathrm{mg}$

Povidone K30

$500 \mathrm{mg}$

Poloxamer 407

$500 \mathrm{mg}$

thereby its oral bio-availability remains one of most challenging aspects of drug development process especially for oral drug delivery system. There are numerous approaches available and reported in literature to enhance the solubility of poorly water soluble drug. The techniques are
Germany), Paraffin liquid (MERCK, India).

\section{METHOD}

Preparation of Solid Dispersion by Solvent Method

At first Spironolactone $(500 \mathrm{mg}$ ) was weighed

Table 2: Formulation for the preparation of Spironolactone solid dispersions by fusion method using different concentration of Poloxamer 407.

\begin{tabular}{llllll}
\hline Ingredients & SPL- Polo A & SPL- Polo B & SPL- Polo C & SPL- Polo D & SPL- Polo E \\
\hline Spironolactone & $500 \mathrm{mg}$ & $500 \mathrm{mg}$ & $500 \mathrm{mg}$ & $500 \mathrm{mg}$ & $500 \mathrm{mg}$ \\
Poloxamer 407 & $200 \mathrm{mg}$ & $400 \mathrm{mg}$ & $600 \mathrm{mg}$ & $800 \mathrm{mg}$ & $1000 \mathrm{mg}$ \\
Acetone & $5 \mathrm{ml}$ & $5 \mathrm{ml}$ & $5 \mathrm{ml}$ & $5 \mathrm{ml}$ & $5 \mathrm{ml}$ \\
\hline
\end{tabular}

chosen on the basis of certain aspects such as properties of drug under consideration, nature of excipients to be selected and nature of intended dosage form. This present work is intended to discuss the various traditional and novel techniques for solubility enhancement of hydrophobic drugs for oral pharmaceutical formulation (Boles Ponto and Schoenwald, 1990; Ozdmir and Ordu, 1998). accurately in an analytical balance and taken into dry and clean glass vials. Then Acetone $(5 \mathrm{ml})$ was added (Patel MM and Patel DM, 2006) on each vial. Then each polymer (500 mg) was dissolved in the solvent using a vortex mixer to make a polymer solution (Table 1). Drug, polymer and solvent combination was dried by using hair dryer until solid dispersion was formed. The solvent 
Table 3: Correlation coefficient $\left(\mathbf{R}^{2}\right)$ values for the formulation of solvent evaporation method.

\begin{tabular}{|c|c|c|c|c|}
\hline \multirow{2}{*}{ Product } & \multirow[b]{2}{*}{ Zero order plot } & \multicolumn{2}{|c|}{ Correlation coefficient $\left(R^{2}\right)$ value } & \multirow[b]{2}{*}{ Higuchi Plot } \\
\hline & & First order Plot & Hixon Crowel Plot & \\
\hline SPL-HPMC & 0.914 & 0.910 & 0.631 & 0.984 \\
\hline SPL-HPC & 0.903 & 0.818 & 0.611 & 0.988 \\
\hline SPL-NaCMC & 0.943 & 0.959 & 0.605 & 0.978 \\
\hline SPL-Povidone K12 & 0.966 & 0.961 & 0.674 & 0.981 \\
\hline SPL-Povidone K30 & 0.928 & 0.895 & 0.621 & 0963 \\
\hline SPL-Poloxamer 407 & 0923 & 0.959 & 0.605 & 0.987 \\
\hline
\end{tabular}

evaporated completely. Finally the formulations were withdrawn from vials, crushed in mortar and pestle, passed through 150 micron sieve. Then the resulted samples were weighed and transferred in fresh vials with proper labeling and its double amount of
Finally the formulations were withdrawn from vials, crushed in mortar and pestle, passed through 150 micron sieve. Then the resulted samples were weighed and transferred in fresh vials with proper labeling and its double

Table 4: Correlation coefficient $\left(\mathbf{R}^{2}\right)$ values for the formulation of fusion method.

\begin{tabular}{lcccc}
\multicolumn{1}{c}{$\begin{array}{c}\text { Product } \\
\text { Formulation }\end{array}$} & Zero order plot & First order Plot & Correlation coefficient $\left(\mathbf{R}^{2}\right)$ value \\
Hixon Crowel Plot & Higuchi Plot \\
\hline SPL-HPMC & 0.904 & 0.920 & 0.601 & 0.981 \\
SPL-HPC & 0.898 & 0.808 & 0.604 & 0.982 \\
SPL-NaCMC & 0.903 & 0.939 & 0.602 & 0.971 \\
& & & & 0.983 \\
SPL-Povidone K12 & 0.906 & 0.901 & 0.607 & 0979 \\
SPL-Povidone K30 & 0.908 & 0.885 & 0.621 & 0.989 \\
SPL-Poloxamer 407 & 0913 & 0.949 & 0.608 & \\
\hline
\end{tabular}

lactose was added on each vials as adsorbent and mixed well. Formulations were kept in desiccators until the dissolution started.

\section{Preparation of Solid Dispersion by Fusion Method}

At first $500 \mathrm{mg}$ of the model drug, Spironolactone was accurately weighted and taken in glass vials and $5 \mathrm{ml}$ Acetone was added as solvent to dissolve each vial and heated on a water bath containing paraffin liquid to melt the ingredients at the temperature of $120^{\circ} \mathrm{C}$. At this time, stirring was performed to make sure a homogenous mixing of the drug in the solvent. When the drug was completely dissolved in the solvent, then $500 \mathrm{mg}$ of each polymer (HPMC 6cps, HPC, NaCMC, Povidone K12, Povidone K30, Poloxamer 407) were weighed accurately and mixed with the solution containing drug and solvent (Table 2). Drug, polymer and solvent combination was cooled with constant stirring to homogeneously disperse the drug throughout the matrix. amount of lactose was added as adsorbent on each vials. After mixing well the formulations were kept in desiccators until the dissolution started.

In vitro dissolution study of Solid Dispersion In-vitro dissolution was carried out in a USP XXX apparatus 2 (Paddle Apparatus) in 900 $\mathrm{ml}$ of distilled water for 1 hour at $37 \pm 0.5^{\circ} \mathrm{C}$ and at a rotational speed of $50 \mathrm{rpm}$. Dissolution samples were withdrawn at predetermined intervals and were filtered through $0.45 \mathrm{~m}$ filters. The drug content was determined spectrophotometrically at $\max =238 \mathrm{~nm}$ in the filtrate either directly or after appropriate dilution with the dissolution media.

\section{RESULT AND DISCUSSION}

Effects of Spironolactone, poorly water soluble drug, Solid Dispersions using various Excipients. After dissolution, we found from the Table1 that the percent release (\%) of drug for seven formulations within 1 hour were $35.27 \%$, 93.05\%, 96.81\%, 89.93\%, $76.17 \%, 67.92 \%$ and $90.84 \%$ of SPL (drug only), SPL-HPMC, SPL-HPC, SPL-NaCMC, 
Figure 1: Effect of different excipients on Spironolactone solid dispersion dissolution rate dissolution condition: distilled water; RPM: $100 ; 37^{\circ} \mathrm{C} \pm 0.5^{\circ} \mathrm{C}$.

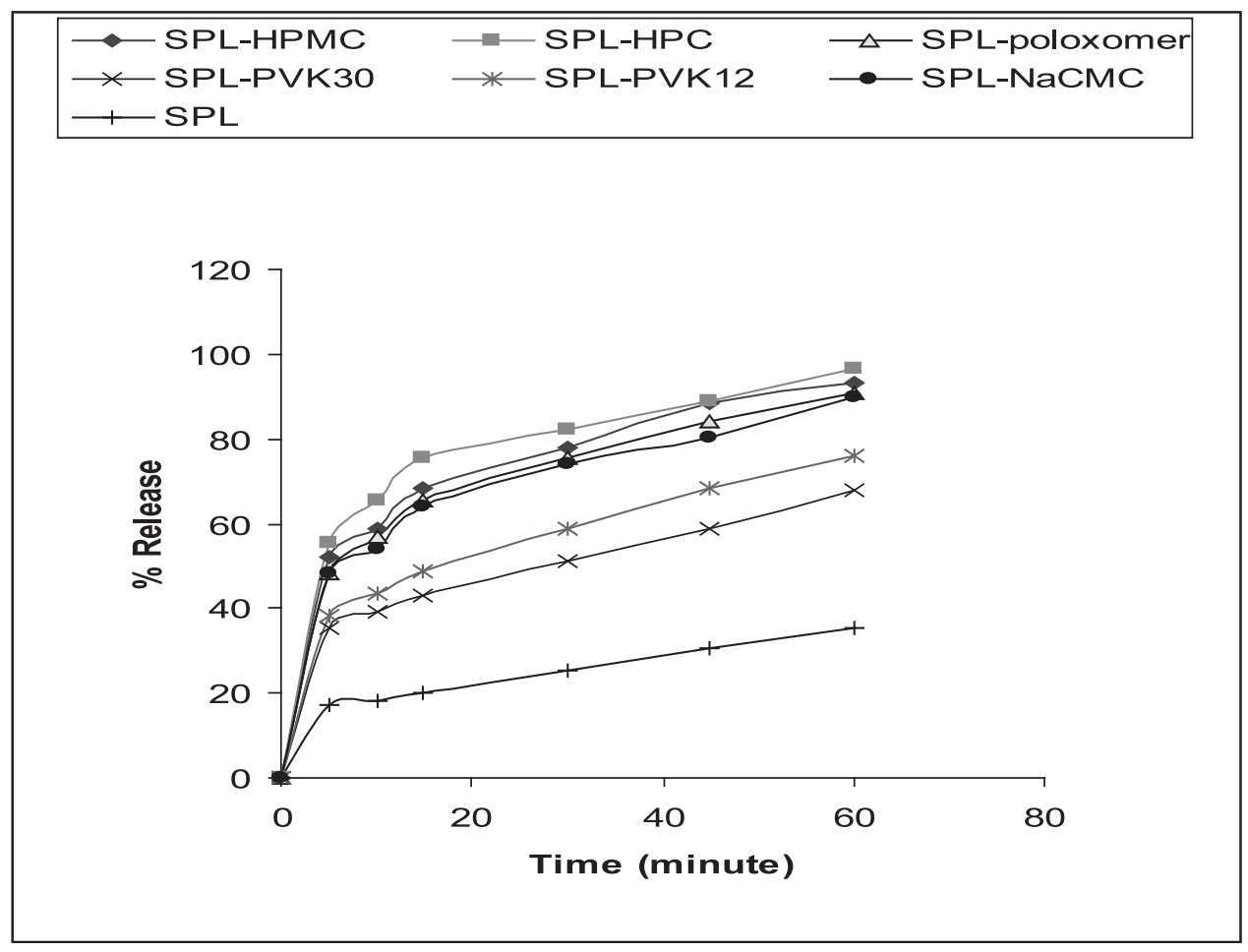

Figure 2: Effect of different concentrations of Poloxamer 407 on Spironolactone solid dispersion dissolution rate dissolution condition: distilled water; RPM: $100 ; 37^{\circ} \mathrm{C} \pm 0.5^{\circ} \mathrm{C}$.

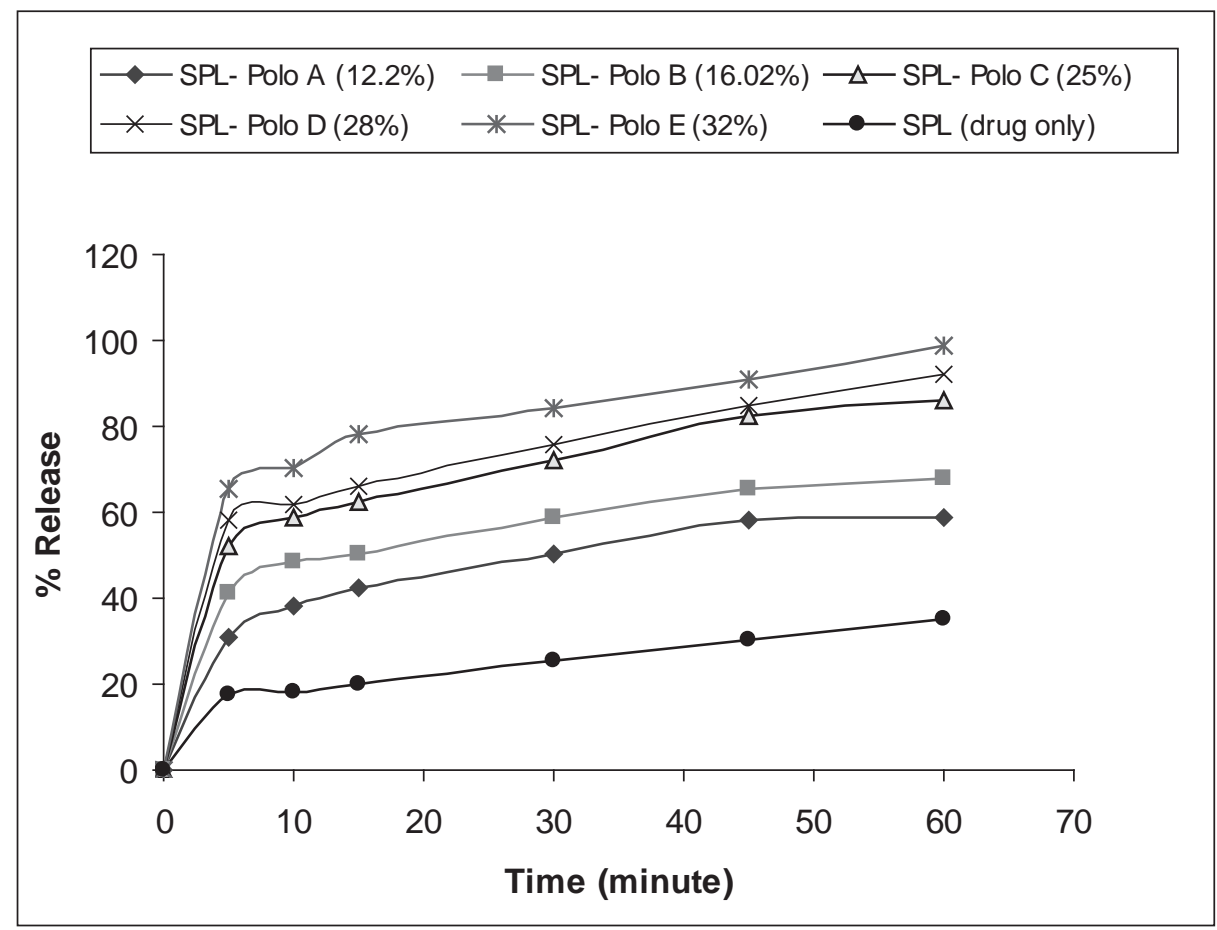


SPL-Povidone K12, SPL-Povidone K30 and SPL-Poloxamer respectively (Figure 1). So the dissolution studies showed the $\%$ release of pure Spironolactone from the solid dispersions was only $35.27 \%$. Here the formulations SPL-HPC, SPL-HPMC, SPLPoloxamer 407, SPL- NaCMC have shown higher $\%$ release respectively.

So it can be said that there was an appeal difference between the release rate of only Spironolactone and solid dispersion containing Spironolactone with different excipients. Solid dispersions containing Spironolactone with HPC, HPMC $6 \mathrm{cp}$, Poloxamer 407 and $\mathrm{NaCMC}$ provided higher release rate than the release rate of solid dispersion containing only Spironolactone, Povidone K12, Povidone K30.

\section{Effects of Spironolactone Solid dispersions using Different Concentrations of Poloxamers 407}

Spironolactone solid dispersions were prepared by fusion method according to Table 2. Here formulations contained drug and different concentrations of Poloxamer 407. The drug and different concentrations of Poloxamer 407 were coded as - SPL-Polo A, SPL-Polo B, SPL-Polo C, SPL-Polo D, SPLPolo $E$ respectively. The release of different concentrations of Poloxamer 407 has shown $12.2 \%, 16.02 \%, 25 \%, 28 \%$ and $32 \%$ of SPLPolo A, SPL-Polo B, SPL-Polo C, SPL-Polo $\mathrm{D}$, SPL-Polo $\mathrm{E}$ respectively. Distilled water was used as dissolution medium. So the dissolution studies showed the percent release of drug for six formulations within 1 hour were $58.80 \%, 67.73 \%, 87.91 \%, 93.42 \%$, $96.16 \%$ and $34.84 \%$ of SPL-Polo A, SPL-Polo B, SPL-Polo C, SPL-Polo D, SPL-Polo E , SPL (drug only) respectively (Figure 2). So the dissolution studies showed the percent release of pure Spironolactone from the solid dispersions was only $34.84 \%$. It can be said that the percent release of drug was increased when increased the concentrations of Poloxomer 407. So, it can be represented as the modified drug release delivery system where Poloxamer 407 was used as the release modifier.

The comparison of percent release between fusion and solvent evaporation method has been shown in Figure 3. The correlation coefficients values of the trend lines of the graphs showed that formulation of Solvent evaporation method (SPL-HPMC, SPL-HPC, SPL-NaCMC, SPL-Povidone K12, SPLPovidone $\mathrm{K} 30$ and SPL-Poloxamer) and Fusion method (SPL-HPMC, SPL-HPC, SPLNaCMC, SPL-Povidone K12, SPL-Povidone $\mathrm{K} 30$ and SPL-Poloxamer) method best fits in Higuchian release pattern. The values of the correlation coefficients (R2) for solvent evaporation method and fusion method have been shown in the Table 3 and Table 4 respectively. It is difficult at this stage to explain in details the actual mechanism of release since, the polymer degradation starts during the dissolution period. However the possible reason of increased dissolution rate of Spironolactone was the use of carriers for which the wettability and spreadability of the precipitated drug occur by reducing aggregations in the readily soluble state.

\section{CONCLUSION}

In this experiment an attempt has been taken to evaluate Spironolactone release from the different polymer-drug loaded formulation of Solid dispersions. Observations of In-vitro dissolution of the solid dispersion of Spironolactone using distilled water as the

Figure 3: Comparison of percent release curve of spironolactone with different formulation methods.

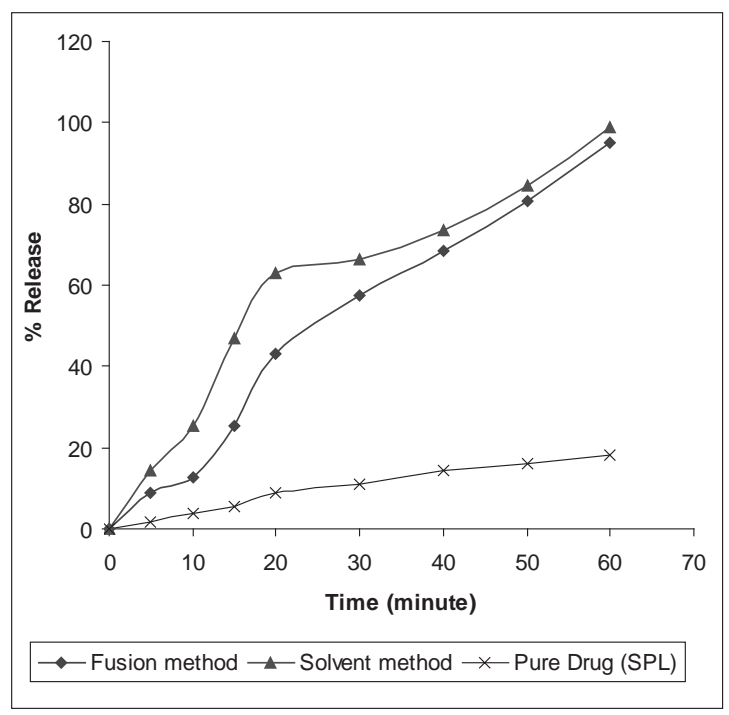


media to study the drug release efficiency from the solid dispersion. These distorted solid dispersions may cause a wide variation in drug release pattern.

Spironolactone is a potassium-sparing diuretic, acts as a competitive antagonist to aldosterone. Spironolactone is poorly watersoluble drug and the present study was aimed to enhance the dissolution property of Spironolactone. So this study was endeavored to observe release pattern of drug from the solid dispersion of Spironolactone by using different polymers, such as HPMC 6cps, HPC, NaCMC, Povidone K12, Povidone K30, Poloxamer 407 The variables affecting drug dissolution was matrix property, hydrophilic excipients loading from the solid dispersion and also depend on the physicochemical property of the drug molecule. The major problem of poorly water soluble drugs especially for new molecules is bioavailability. Thus the main target is to increase the solubility of poorly water soluble drugs. So the present study reveals that solid dispersion may be an ideal means of drug delivery system for poorly water soluble drugs. Further study in this field is required to establish these drug delivery systems so that in future it can be used effectively in commercial basis.

\section{REFERENCES}

Babu GVMM, Kumar NR, Himasankar K, Seshasayana A, Murthy KV (2003). Nimesulide-modified gum karaya solid mixtures: preparation, characterization and formulation development. Drug Dev Ind Pharm. 29: 855864. http://dx.doi.org/10.1081/DDC-120024181 PMid:14570306

Babu GVMM, Prasad CHDS, Murthy KVR (2002). Evaluation of modified gum karaya as carrier for the dissolution enhancement of poorly water soluble drug nimodipine. Int J Pharm. 234:117.

http://dx.doi.org/10.1016/S0378-5173(01)00925-5

Boles Ponto LL, Schoenwald RD (1990). Furosemide: a pharmacokinetic / pharmacodynamic review part I. Clin. Pharmacokinet. 18: 381 - 408.

http://dx.doi.org/10.2165/00003088-199018050-00004

Chiou WL, Riegelman S (1971). Pharmaceutical applications of solid dispersion systems. J Pharm Sci. 60: 1281-1302. http://dx.doi.org/10.1002/jps.2600600902 PMid:4935981
El-Gazayerly ON. (2000) Characterization and evaluation of tenoxicam coprecipitates. Drug Dev Ind Pharm. 26: 925-930. http://dx.doi.org/10.1081/DDC-100101319 PMid:10914316

Hirasawa NI, Shise S, Miyata H, Danjo K (2009). Physiochemical characteristics and drug release studies of nilvadipine solid dispersions using Chaulang. J Pharm Res. 8 (1): 51

Loganathan S, Maimaran S, Rajasekaran A, Reddy MVP, Sulaiman $A$ (2000). The effect of solid dispersions on (solubility) dissolution rate of ibuprofen. The Eastern Pharmacist. 513: 115 - 116.

Makiko F, Hideko O, Yu suke S, Honami T, Masuo K, Yoshiteru W (2005). Preparation, characterization, and tableting of a solid dispersion of indomethacin with crospovidone. Int. J. Pharm. 293: 145-153. http://dx.doi.org/10.1016/j.ijpharm.2004.12.018 PMid:15778052

Murray MD, Haag KM, Black PK, Hall SD, Brater DC (1997). Variable furosemide absorption and poor predictability of response in elderly patient.

Pharmacotherapy. 17: 98 - 106. PMid:9017769

Ozdmir N, Ordu S (1998). Improvement of dissolution properties of furosemide by complexation with cyclodextrin. Drug Dev. Ind. Pharm. 24 (1): 19 - 25. http://dx.doi.org/10.3109/03639049809082348 PMid:15605593

Patel MM, Patel DM (2006). Fast dissolving Valdecoxib tablets containing solid dispersion of Valdecoxib. Indian Journal of Pharmaceutical Science. 68(2): 222-226. http://dx.doi.org/10.4103/0250-474X.25719

Rogers JA, Anderson AJ (1982). Physical characteristics and dissolution profiles of ketoprofen-urea solid dispersions. Pharm Acta Helv. 57: 276-281.

Vippagunta SR, Maul KA, Tallavajhala S, Grant DJW. (2002) Solid-state characterization of nifedipine solid dispersions. Int J Pharm. 236: 111-123. http://dx.doi.org/10.1016/S0378-5173(02)00019-4

Yamashita K, Nakate T, Okimoto KA. (2003)

Establishment of new preparation method for solid dispersion formulation of tacrolimus. Int J Pharm. 267: 79-91. http://dx.doi.org/10.1016/j.ijpharm.2003.07.010 PMid:14602386 